\title{
ANÁLISES FAUNÍSTICA E MULTIVARIADA DE FORAMINÍFEROS PLANCTÔNICOS DA BACIA PERNAMBUCO-PARAÍBA: UMA INTERPRETAÇÃO PALEOAMBIENTAL DOS ÚLTIMOS 30 MIL ANOS
}

\author{
EDMUNDO CAMILLO JR., JULIANA PEREIRA DE QUADROS, \\ KAREN BADARACO COSTA \& FELIPE ANTONIO DE LIMA TOLEDO \\ Laboratório de Paleoceanografia do Atlântico Sul - LaPAS, Instituto Oceanográfico, Universidade de São Paulo, \\ Praça do Oceanográfico, 191, 05508-900, São Paulo, SP. edmundo@paleolapas.org, \\ juliana@paleolapas.org,karen.costa@usp.br,ftoledo@usp.br
}

\begin{abstract}
ABRSTRACT-FAUNALAND MULTIVARIATEANALYSES OF PLANKTONIC FORAMINIFERAFROM PERNAMBUCOPARAIIBA BASIN: A PALEOENVIRONMENTAL INTERPRETATION FOR THE LAST $30 \mathrm{KYR}$. This study investigated the planktonic foraminifera of 23 samples from two sediment cores of the continental slope (between 1,260 and 1,315 m deep) of Pernambuco-Paraíba Basin (Brazil), western South Atlantic. For each sample, 300-600 specimens of foraminifers were identified. The relative abundance of species was calculated and then employed to statistical analysis. Recovered planktonic foraminifera assemblages represent a typical tropical association involving species of warm water, especially Globigerinoides ruber and Globigerinoides sacculifer. This dominance makes difficult the recognition of the relationship between environmental parameters and changes in abundance of other species. The results indicate relative stability of general environmental conditions over the last 30,000 years in the study area. The application of Q-mode factor analysis helped in the identification of the most significant factors for the planktonic foraminifera. Changes in the mixed layer and the temperature of the sea surface were considered the main environmental parameters that can influence the distribution of planktonic foraminifera recorded in the Pernambuco-Paraíba Basin. We believe that the dynamics of winds related to the Intertropical Convergence Zone influences the study area, both for charging the higher temperatures of the sea surface, as well as promoting greater or lesser vertical mixing of the ocean in this region.
\end{abstract}

Key words: planktonic foraminifera, Pernambuco-Paraíba Basin, factorial analysis.

RESUMO - O presente estudo investigou os foraminíferos planctônicos de 23 amostras provenientes de dois testemunhos de sedimento do talude continental (entre 1.260 e $1.315 \mathrm{~m}$ de profundidade) da bacia Pernambuco-Paraíba (Brasil), porção oeste do Atlântico Sul. Foram identificados entre 300-600 espécimes de foraminíferos por amostra. A abundância relativa das espécies foi calculada e então empregada no tratamento estatístico. As assembleias de foraminíferos planctônicos recuperadas representam uma típica associação tropical caracterizada por espécies de água quente, especialmente Globigerinoides ruber e Globigerinoides sacculifer. Esta dominância dificulta o reconhecimento da relação existente entre os parâmetros ambientais e as variações de abundância das demais espécies. Os resultados deste estudo indicaram uma relativa estabilidade das condições ambientais gerais ao longo dos últimos 30.000 anos na área de estudo. A aplicação da análise fatorial modo-Q auxiliou na identificação dos fatores mais significativos para os foraminíferos planctônicos. As variações na camada de mistura e na temperatura da superfície do mar foram consideradas como os principais parâmetros ambientais capazes de influenciar a distribuição dos foraminíferos planctônicos registrados na bacia Pernambuco-Paraíba. Acreditamos que a dinâmica de ventos relacionada à Zona de Convergência Intertropical influencia a região estudada, tanto por conduzir as maiores temperaturas da superfície do mar, como também por promover maior ou menor mistura nesta região do oceano.

Palavras-chave: foraminíferos planctônicos, bacia Pernambuco-Paraíba, análise fatorial.

\section{INTRODUÇÃO}

Diversas espécies de foraminíferos planctônicos reagem sensivelmente a diferentes fatores oceanográficos como, por exemplo, profundidade da camada de mistura, profundidade da termoclina, condições de ressurgência, disponibilidade de nutrientes e oxigenação (Hilbretch, 1996). Apesar desta complexidade, assume-se que todas as espécies contribuem com alguma informação relacionada à temperatura da água na qual viveram e se reproduziram (Kemle-von Mücke \& Oberhänsli, 1999).

Alguns autores, entretanto, sugerem que variações na abundância relativa das espécies ao longo de uma série temporal podem ser melhor explicadas pela mudança na profundidade da termoclina do que exclusivamente pela temperatura (Molfino et al., 1982; Ravelo et al., 1990; Andreasen \& Ravelo, 1997; Chen \& Prell, 1998). Estas particularidades envolvendo os foraminíferos planctônicos, 
combinadas ao fato de que quase todas as espécies modernas passam parte de seu ciclo de vida nas camadas mais superficiais, dentro da zona fótica, fazem deste grupo de organismos um excelente instrumento no estudo das condições superficiais do passado (Oberhänsli et al., 1992; Kemle-von Mücke \& Oberhänsli, 1999).

Nos estudos de caráter paleoceanográfico e paleoecológico, as assembleias microfossilíferas são selecionadas de diferentes localidades e/ou intervalos de tempo. Isto requer uma análise e uma interpretação de dados compostos por grande número de variáveis (Prentice, 1986). Diversos estudos demonstraram a importância das abordagens estatísticas no auxílio às interpretações de assembleias microfossilíferas (e.g. Toledo et al., 1999; Jayalakshmy \& Rao, 2003; Buccianti \& Esposito, 2004; Hirama et al., 2010; Gandhi \& Solai, 2010; Oláyíwolá \& Odébòdé, 2011; Guerreiro et al., 2011). Desta forma, a análise estatística multivariada é importante, já que ressalta os parâmetros ecológicos dominantes, simplificando a estrutura dos dados, tornando-os mais fáceis de serem explorados (Kucera \& Malmgren, 1998; Parker \& Arnold, 1999; Gandhi \& Solai, 2010).

Para entender as condições oceanográficas na região equatorial brasileira durante os últimos 30.000 anos, este estudo pretende explorar a distribuição dos foraminíferos planctônicos em dois testemunhos marinhos da margem continental nordeste do Brasil. Estes dados quantitativos foram submetidos à análise fatorial modo-Q como procedimento de identificação de parâmetros oceanográficos que atuaram com maior ou menor intensidade ao longo do tempo (Toledo et al., 1999). Desta forma, tornou-se possível relacionar as oscilações na abundância relativa das principais espécies de foraminíferos planctônicos ao longo do testemunho com as mudanças paleoceanográficas/paleoclimáticas ocorridas nos últimos 30.000 anos.

\section{MATERIAL E MÉTODOS}

\section{Testemunhos}

Neste estudo foram utilizados dois testemunhos provenientes do talude continental da bacia PernambucoParaíba, localizada na margem continental nordeste do Brasil (Figura 1). Esta região está situada dentro da Zona de Convergência Intertropical que se caracteriza, especialmente, pelo controle dos ventos alísios sobre as correntes superficiais no Atlântico tropical. A ação direta dos ventos sobre a camada superficial marinha induz os processos de mistura estabelecendo a camada de mistura que, naquela região, ultrapassa $150 \mathrm{~m}$ (WOA98). De acordo Hüttl \& Böning (2007), na porção leste do Atlântico Sul a termoclina é mais rasa e íngreme, enquanto que na porção oeste a camada de mistura é mais profunda e a termoclina está abaixo da zona fótica. Segundo os autores, variações na intensidade dos ventos alísios exercem controle sobre as variações na intensidade de ressurgências e downwelling e, desta forma, na profundidade da camada de mistura e da termoclina.

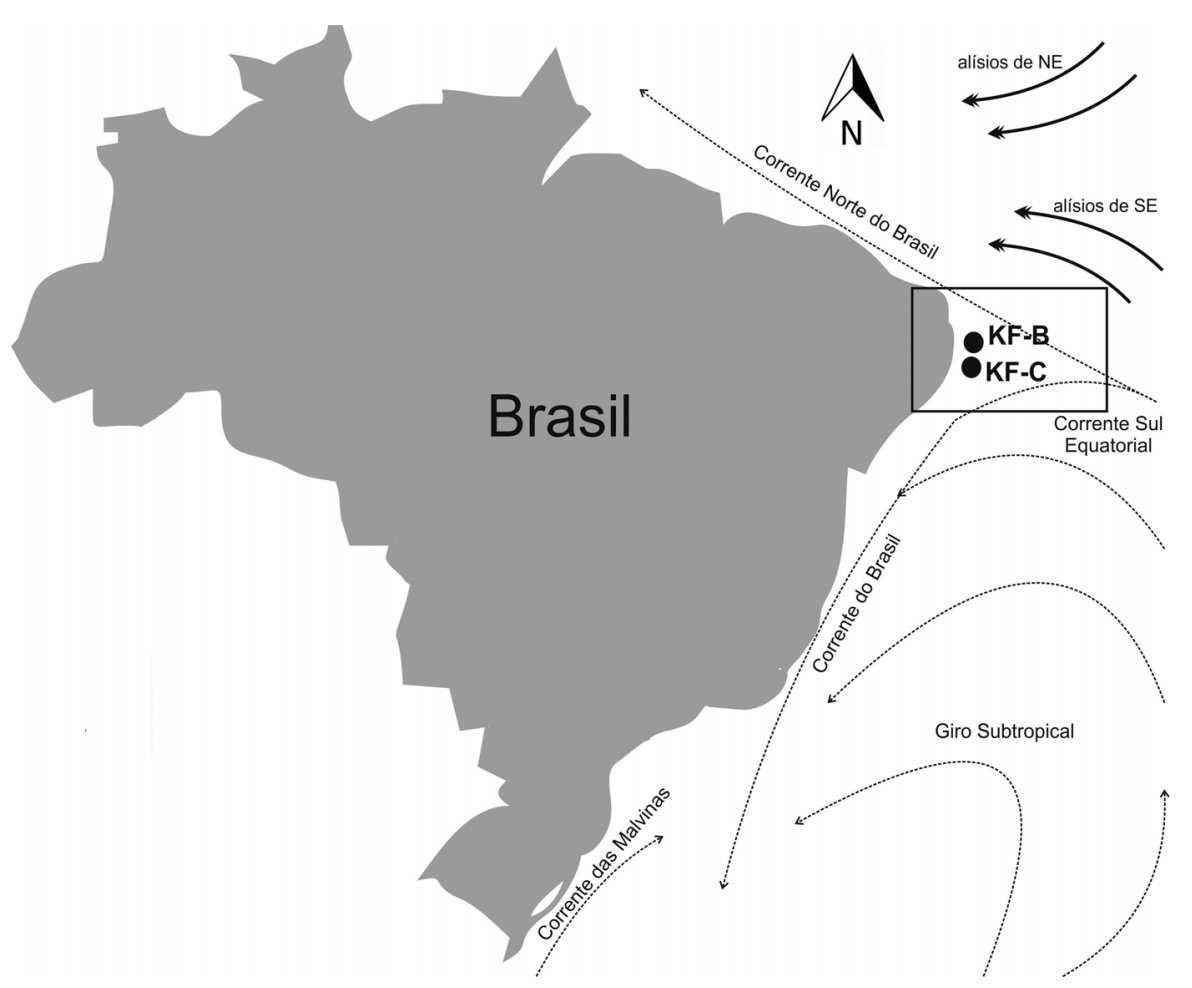

Figura 1. Representação esquemática da circulação superficial de larga-escala no Atlântico Sul e localização dos testemunhos estudados (modificado de Peterson \& Stramma, 1991).

Figure 1. Schematic map of the large-scale upper-level circulation in the South Atlantic and the studied cores (modified from Peterson \& Stramma, 1991). 
Os testemunhos KF-C e KF-B (Tabela 1) utilizados neste estudo, foram retirados de profundidades acima da lisoclina moderna como proposto por Broecker \& Takahashi (1978), o que minimiza os efeitos de dissolução no material micropaleontológico. Os testemunhos foram coletados a pistão pela Texas University, entre 26 de janeiro e 5 de março de 1996, a bordo do R/V Powell, em uma campanha de prospecção geoquímica nas bacias da costa brasileira coordenada pelo Centro de Pesquisas da Petrobras - Cenpes. $O$ estudo sedimentológico e $\mathrm{o}$ arquivamento foram realizados pelo Laboratório de Rocha da Gerência de Exploração da bacia de Campos. Os procedimentos de descrição e amostragem dos testemunhos estão descritos em Toledo (2000) e Costa (2000).

\section{Análise faunística de foraminíferos planctônicos}

As amostras aqui utilizadas foram lavadas em peneiras de malha de 0,062 $\mathrm{mm}$ para a separação das frações finas e grossas, preservando-se ambas as frações. Em seguida as amostras foram secas em estufa à temperatura inferior a $60^{\circ} \mathrm{C}$. A fração grossa foi peneirada novamente, desta vez fazendo uso de peneiras com malhas de $0,150 \mathrm{~mm}$, com a qual foram realizadas as contagens da fauna e a análise isotópica de foraminíferos planctônicos. A fração retida na malha de $0,150 \mathrm{~mm}$ foi dividida até que se atingisse entre 300-600 espécimens contabilizados para, a partir de então, efetuar a análise quantitativa da fauna. A identificação dos foraminíferos planctônicos presentes neste estudo segue principalmente Hemleben et al. (1989) e, em alguns casos, também Bolli \& Saunders (1985).

\section{Análise fatorial modo-Q}

A técnica de análise fatorial modo-Q (rotação Varimax) foi aplicada sobre os dados de abundância relativa dos dois testemunhos estudados, obtidos da análise faunística. Tradicionalmente, espécies com abundâncias relativas abaixo de 1\% são eliminadas. Entretanto, é importante esclarecer aqui que os resultados analíticos não tratarão destas espécies ou da porção da fauna que elas representam (Parker \& Arnold, 1999; Buccianti \& Esposito, 2004). A análise fatorial foi executada no programa Statistica 9.

A análise fatorial assume que a informação contida nos dados é composta de poucos fatores comuns a todas as entidades (amostras), somada aos fatores particulares de cada entidade individual (espécie). Os fatores fundamentais comuns não são diretamente observados, mas são refletidos

Tabela 1. Localização, profundidade e comprimento dos testemunhos utilizados neste estudo.

Table 1. Location, depth and recovered length of the studied cores.

\begin{tabular}{lcc}
\hline & Testemunho KF-B & Testemunho KF-C \\
\hline Localização & $07^{\circ} 29^{\prime} 37.0^{\prime \prime} \mathrm{S} /$ & $08^{\circ} 22^{\prime} 9,78^{\prime} \mathrm{S} /$ \\
& $34^{\circ} 20^{\prime} 27.1^{\prime \prime} \mathrm{W}$ & $34^{\circ} 27^{\prime} 49,68^{\prime \prime} \mathrm{W}$ \\
Profundidade de amostragem & $1261 \mathrm{~m}$ & $1315 \mathrm{~m}$ \\
Comprimento recuperado & $398 \mathrm{~cm}$ & $487 \mathrm{~cm}$ \\
Comprimento utilizado & $19 \mathrm{~cm}$ & $114 \mathrm{~cm}$ \\
\hline
\end{tabular}

nas entidades observadas. Uma implicação da abordagem da análise fatorial é que o número de fatores fundamentais comuns pode ser conhecido facilitando a interpretação ambiental (Toledo, 2000; Jayalakshmy \& Rao, 2003).

\section{Cronologia e seleção das amostras}

A cronologia para os testemunhos foi estabelecida a partir de análises de isótopos de oxigênio em foraminíferos planctônicos (Globigerinoides ruber) para os dois testemunhos estudados. Além disso, foram efetuadas datações de radiocarbono $\left({ }^{14} \mathrm{C}\right) \mathrm{em}$ quatro amostras, duas por testemunho. As análises de isótopos estáveis de oxigênio e de carbono, bem como as datações de radiocarbono foram efetuadas no National Ocean Science Accelerator Mass Spectrometer Facility (NOSAMS) do Woods Hole Oceanographic Institution (WHOI), EUA. Com estes dados foi estabelecido um modelo de idade pelo ajuste entre as curvas isotópicas deste estudo e a curva de referência LR04 (Lisiecki \& Raymo, 2005).

As idades de radiocarbono foram corrigidas para efeito reservatório de acordo com Butzin et al. (2005). A conversão para idades calendário foi efetuada com base na curva de calibração proposta por Fairbanks et al. (2005). Este estudo optou pelo modelo de Butzin et al. (2005) para correção da idade reservatório, já que Fairbanks et al. (2005) consideram este o melhor modelo disponível sobre a estimativa de idades reservatório dos oceanos modernos.

De acordo com o modelo de Butzin et al. (2005), a idade reservatório nesta área de estudo é menor que o padrão até então utilizado para as regiões tropicais, de 400 anos em média. As idades reservatório sugeridas pelos autores e utilizadas para a correção foram 288 anos. As correções para efeito reservatório e as conversões para idade calendário são mostradas na Tabela 2 .

Para este estudo foram selecionadas 15 amostras do testemunho KF-C $(114 \mathrm{~cm})$ e oito do KF-B $(19 \mathrm{~cm})$, pois de acordo com os resultados isotópicos $\left(\delta^{18} \mathrm{O}\right)$ e bioestratigráficos estas amostras seriam suficientes para compreender o período de interesse, i.e., os últimos 30.000 anos. $\mathrm{O}$ intervalo de amostragem foi de aproximadamente $11 \mathrm{~cm}$ no testemunho KF-C e mais detalhado no KF-B, cerca de $3 \mathrm{~cm}$ entre cada amostra.

Tabela 2. Datação de radiocarbono, correção para efeito reservatório e conversão para idade calendário das quatro amostras datadas. Abreviaturas: D.P., desvio padrão; I.R., idade reservatório; I.C., idade corrigida; I.Cal., idade calendário.

Table 2. Radiocarbon dating, reservoir effect correction and conversion to calendar ages. Abbreviations: D.P., standard deviation; I.R., reservoir age; I.C., corrected age; I.Cal., calendar age.

\begin{tabular}{ccccccc}
\hline Testemunho & $\begin{array}{c}\text { Amostra } \\
(\mathrm{cm})\end{array}$ & Idade & d.p. & I. R. & I. C. & I. Cal. \\
\hline KF-C & 81 & 17.500 & 80 & 288 & 17.212 & $20.337 \pm 74$ \\
KF-C & 159 & 38.900 & 330 & 288 & 38.612 & $42.960 \pm 287$ \\
KF-B & 3,5 & 5.890 & 35 & 288 & 5.602 & $6.379 \pm 40$ \\
KF-B & 19 & 32.100 & 180 & 288 & 31.812 & $36.774 \pm 124$ \\
\hline
\end{tabular}




\section{RESULTADOS}

\section{Modelo de idade $\left(\delta^{18} \mathrm{O}\right.$ vs. LR04)}

O modelo de idade dos testemunhos estudados (Tabela 3) foi estabelecido com base no ajuste entre as curvas isotópicas de oxigênio e a curva de referência LR04 (Lisiecki \& Raymo, 2005). As duas datações por testemunho foram utilizadas como pontos de controle do modelo. Os resultados dos valores dos isótopos estáveis de oxigênio estimados para os dois testemunhos estudados a partir da testa do foraminífero planctônico Globigerinoides ruber 'branca' são apresentados na Tabela 4.

De acordo com os dados percentuais do plexo Globorotalia menardii, da estratigrafia isotópica do oxigênio (Figura 2) e das datações de radiocarbono, foi possível reconhecer que ambos os testemunhos estudados alcançaram o último período glacial (Estágio Isotópico Marinho - EIM - 2) assim como a Biozona Y de Ericson \& Wollin (1968). No testemunho KF$\mathrm{C}$, de maior resolução e taxa de sedimentação, também foi evidente o reconhecimento do período interglacial atual (EIM 1, equivalente ao Holoceno) bem como da Biozona $\mathrm{Z}$ de Ericson \& Wollin (1968). No entanto, no testemunho KF-B apenas duas amostras são representativas deste período (EIM 1).

Tabela 3. Idades calendário dos testemunhos KF-C e KF-B. As amostras em destaque $\left({ }^{\bullet}\right)$ foram datadas e as demais interpoladas (interpolação linear).

Table 3. KF-C and KF-B calendar ages. Samples identified with ( $\left(^{\bullet}\right)$ were dated and the others were interpolated (linear interpolation).

\begin{tabular}{|c|c|}
\hline \multicolumn{2}{|c|}{ Testemunho KF-C } \\
\hline Amostra (cm) & Idade (k.a.) \\
\hline 0 & 0,006 \\
\hline 11 & 2,8 \\
\hline 23 & 5,8 \\
\hline 35 & 8,8 \\
\hline 46 & 11,6 \\
\hline 53 & 13,3 \\
\hline 57 & 14,3 \\
\hline 61 & 15,3 \\
\hline 65 & 16,3 \\
\hline 69 & 17,3 \\
\hline 74 & 18,6 \\
\hline • 81 & 20,3 \\
\hline 92 & 23,5 \\
\hline 103 & 26,7 \\
\hline 114 & 29,9 \\
\hline \multicolumn{2}{|c|}{ Testemunho KF-B } \\
\hline 1 & 1,8 \\
\hline - 3,5 & 6,4 \\
\hline 6 & 11,3 \\
\hline 8,5 & 16,2 \\
\hline 10,5 & 20,1 \\
\hline 12,5 & 24 \\
\hline 15 & 29 \\
\hline - 19 & 36,8 \\
\hline
\end{tabular}

Tabela 4. Valores de $\partial^{18} \mathrm{O}$ em Globigerinoides ruber 'branca' para os dois testemunhos estudados.

Table 4. Values of $\partial^{18} \mathrm{O}$ in Globigerinoides ruber 'white' for the two studied cores.

\begin{tabular}{cc}
\hline \multicolumn{3}{c}{ Testemunho KF-C } \\
\hline Prof. $(\mathrm{cm})$ & $\partial^{18} \mathrm{O}$ \\
\hline 0 & $-1,7$ \\
11 & $-1,7$ \\
23 & $-1,4$ \\
35 & $-1,4$ \\
46 & $-1,3$ \\
53 & $-1,2$ \\
57 & $-1,2$ \\
61 & $-1,3$ \\
65 & $-1,1$ \\
69 & $-1,0$ \\
74 & $-0,7$ \\
81 & $-0,7$ \\
92 & $-0,8$ \\
103 & $-0,7$ \\
114 & $-0,8$ \\
\hline & \\
1 & Testemunho KF-B \\
3,5 & $-1,4$ \\
6 & $-1,1$ \\
8,5 & $-0,8$ \\
10,5 & $-0,7$ \\
12,5 & $-0,7$ \\
15 & $-0,4$ \\
19 & $-0,8$ \\
\hline & $-0,6$ \\
\hline & \\
\hline
\end{tabular}

\section{Análise faunística}

As principais espécies observadas, i.e., as espécies mais abundantes e frequentes, estão apresentadas nas tabelas 5 e 6 . As assembleias são distintamente tropicais dominadas por espécies de águas quentes, especialmente Globigerinoides ruber e G. sacculifer. Mesmo com espécies dominantes, os foraminíferos planctônicos das amostras estudadas apresentaram um padrão de distribuição complexo, sem relação evidente com os principais fatores ambientais. Destacaram-se no testemunho KF-C: G. ruber 'branca' (37,5\%-67,5\%), G. ruber 'rosa' (1,1\%-10,5\%), G. sacculifer s/saco (13,7\%-26,5\%) e G. sacculifer c/saco (0,8\%-5,1\%); seguidas por $G$. rubescens $(1,2 \%-10,8 \%), G$. siphonifera (1,6\%-8,3\%), G. falconensis $(0-5,8 \%)$, G. calida $(0-4,6 \%)$, G. glutinata (1\%-3,4\%), C. nitida (0-3,2\%), e G. bulloides (0-1,5\%). No testemunho KF-B dominaram G. ruber 'branca' (33,7\%-52,7\%), G. ruber 'rosa' (11,3\%-17,8\%), G. sacculifer sem saco (5,1\%-21,1\%) e G. sacculifer com saco (4,1\%-10,4\%); além das espécies G. siphonifera $(1 \%$ 4,4\%), G. calida (0,6\%-5,6\%), G. rubescens $(0,2 \%-2,5 \%)$, G. falconensis (1,7\%-3,4\%), G. bulloides (0,2\%-1,4\%), G. glutinata $(0,6 \%-3,1 \%)$ e C. nitida $(0-1,9 \%)$.

A espécie Globigerinoides ruber 'branca' foi predominante ao longo de todas as amostras observadas nos dois 

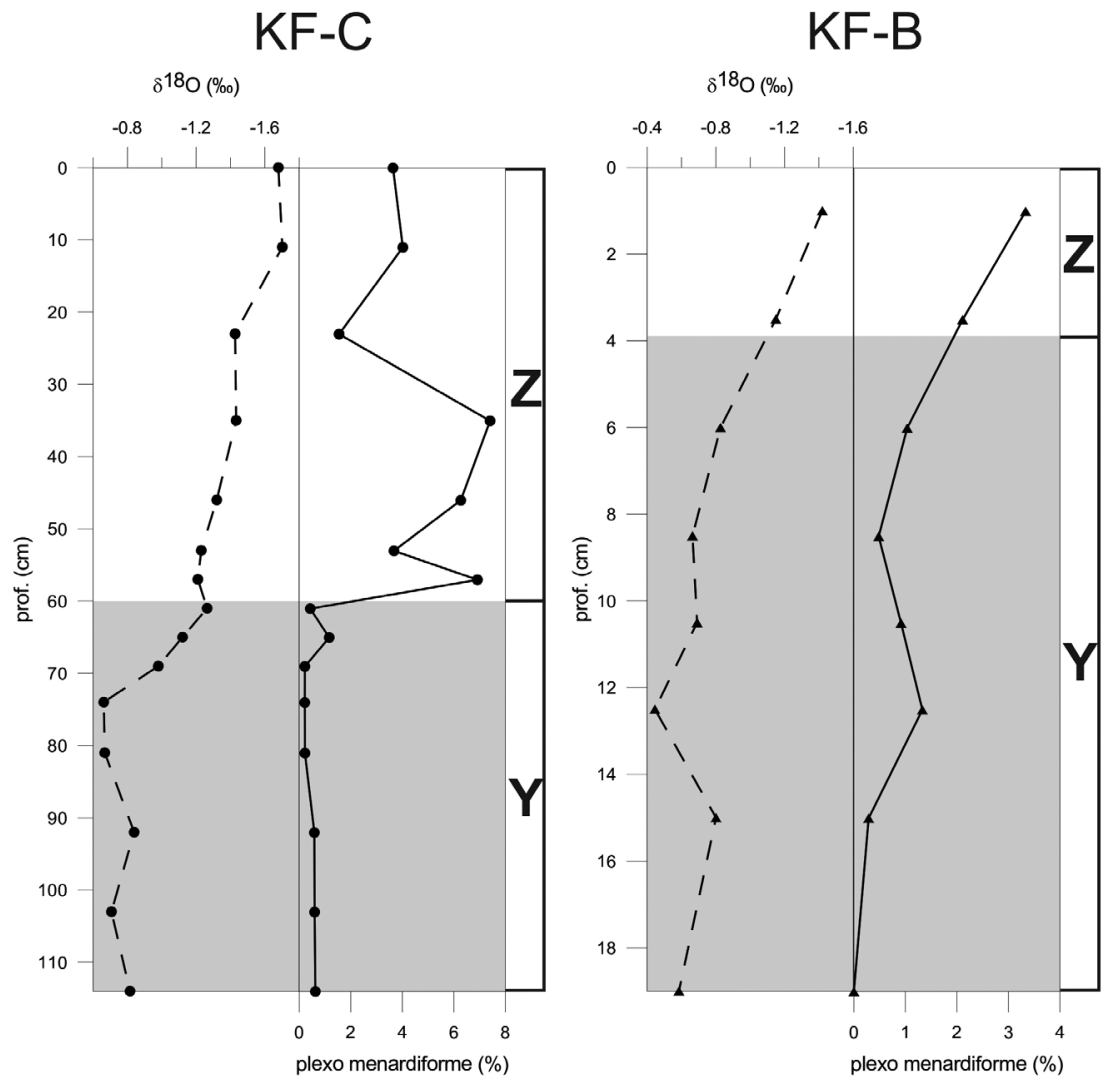

Figura 2. Curva isotópica de oxigênio $\left(\delta^{18} \mathrm{O}\right)$ a partir do foraminífero planctônico Globigerinoides ruber 'branca' e a abundância relativa do plexo Globorotalia menardii ao longo dos testemunhos KF-C (à esquerda) e KF-B (à direita) nos últimos 30 mil anos.

Figure 2. Oxygen isotopic curve in Globigerinoides ruber 'white' and relative abundance of Globorotalia menardii complex along KF-C (left) and KF-B (right) during the last $30 \mathrm{kyr}$.

Tabela 5. Percentuais das principais espécies de foraminíferos planctônicos observadas no testemunho KF-C.

Table 5. Minimum and maximum abundance (\%) of the main species of planktonic foraminifera in the core KF-C.

\begin{tabular}{lcc}
\hline \multicolumn{3}{c}{ Testemunho KF-C } \\
\hline Espécies & Mínimo (\%) & Máximo (\%) \\
\hline G. ruber (var. branca) & 37,5 & 67,5 \\
G. ruber (var. rosa) & 1,1 & 10,5 \\
G. sacculifer (s/ saco) & 13,7 & 24,1 \\
G. sacculifer (c/ saco) & 0,8 & 5,1 \\
G. rubescens & 1,2 & 10,8 \\
G. siphonifera & 1,6 & 6,6 \\
G. calida & 0 & 4,6 \\
G. falconensis & 0 & 5,8 \\
G. bulloides & 0 & 1 \\
G. glutinata & 1 & 3,4 \\
C. nitida & 0 & 3,2 \\
G. menardii & 0 & 3,9 \\
G. tumida & 0 & 2,9 \\
G. tumida flexuosa & 0 & 2,9 \\
G. truncatulinoides (dex. + sin.) & 0 & 6,2 \\
Pulleniatina sp. & 0 & 1,4 \\
\hline
\end{tabular}

Tabela 6. Percentuais das principais espécies de foraminíferos planctônicos observadas no testemunho KF-B.

Table 6. Minimum and maximum abundance (\%) of the main species of planktonic foraminifera in the core KF-B.

\begin{tabular}{lcc}
\hline \multicolumn{3}{c}{ Testemunho KF-B } \\
\hline Espécies & Mínimo (\%) & Máximo (\%) \\
\hline G. ruber (var. branca) & 33,7 & 52,7 \\
G. ruber (var. rosa) & 11,3 & 17,8 \\
G. sacculifer (s/ saco) & 5,1 & 21,1 \\
G. sacculifer (c/ saco) & 4,1 & 10,4 \\
G. rubescens & 0,2 & 2,5 \\
G. siphonifera & 1 & 4,4 \\
G. calida & 0,6 & 5,6 \\
G. falconensis & 1,7 & 3,4 \\
G. bulloides & 0,2 & 1,4 \\
G. glutinata & 0,6 & 3,1 \\
C. nitida & 0 & 1,9 \\
G. menardii & 0 & 0,8 \\
G. tumida & 0 & 1,8 \\
G. tumida flexuosa & 0 & 1,1 \\
G. truncatulinoides (dex. + sin.) & 0 & 2,8 \\
Pulleniatina sp. & 0 & 1,9
\end{tabular}


testemunhos estudados. Porém no testemunho KF-C são observadas duas quedas significativas na abundância relativa desta espécie, em 17.300 e em 13.300 anos, onde foi observada a menor abundância desta espécie neste testemunho. Coincidentemente, foram nesses dois momentos que G. sacculifer 's/ saco' exibiu os maiores valores de sua abundância relativa no KF-C. Quanto às demais espécies da assembleia os dados são difusos, dificultando, assim, o entendimento da relação entre a variação de abundância de cada espécie e os parâmetros ambientais atuantes nessa região do oceano.

\section{Análise fatorial}

Testemunho KF-C. A análise fatorial modo-Q para os dados do testemunho KF-C extraiu 15 fatores, cujas variâncias acumuladas atingiram $100 \%$ (Tabela 7). No entanto, apenas o fator 1 exibiu autovalor maior que 1,0 e, sozinho, é suficiente para explicar 97,98\% da variabilidade da assembleia faunística. Assim, o fator 1 deve ser interpretado como a principal influência na assembléia dos foraminíferos planctônicos durante os últimos 30.000 anos. O fator 2 apresentou um autovalor menor que 1,0, porém este também foi considerado na interpretação paleoceanográfica. Juntos, os fatores 1 e 2 explicam 99,11\% da variância total.

De acordo com os dados da matriz de escores (Tabela 8), que aponta o quanto cada espécie contribuiu para cada um dos fatores extraídos, observa-se que o fator 1 é dominado pela espécie Globigerinoides ruber 'branca' $(4,8456)$. Para o fator 2 verificam-se os maiores escores para Globigerinoides sacculifer 's/ saco' $(4,2933)$. A Tabela 9 exibe as comunalidades e as cargas fatoriais dos fatores $1 \mathrm{e} 2$. As comunalidades indicam a eficiência das cargas fatoriais na explicação da variabilidade total, e as cargas fatoriais

Tabela 7. Fatores, autovalores e suas variâncias para o testemunho KF-C.

Table 7. Factors, eigenvalues and total variance for the core KF-C.

\begin{tabular}{cccc}
\hline \multicolumn{3}{c}{ Testemunho KF-C } \\
\hline Fatores & Autovalor & Variância (\%) & Variância total acumulada (\%) \\
\hline 1 & 14,7 & 97,98 & 97,98 \\
2 & 0,17 & 1,12 & 99,11 \\
3 & 0,05 & 0,36 & 99,47 \\
4 & 0,03 & 0,22 & 99,71 \\
5 & 0,02 & 0,13 & 99,83 \\
6 & 0,008 & 0,06 & 99,89 \\
7 & 0,005 & 0,03 & 99,92 \\
8 & 0,004 & 0,02 & 99,95 \\
9 & 0,003 & 0,02 & 99,97 \\
10 & 0,002 & 0,01 & 99,98 \\
11 & 0,001 & 0,007 & 99,98 \\
12 & 0,0008 & 0,005 & 99,99 \\
13 & 0,0006 & 0,004 & 99,99 \\
14 & 0,0003 & 0,002 & 99,99 \\
15 & 0,0001 & 0,0007 & 100 \\
\hline
\end{tabular}

Tabela 8. Matriz de escores com a contribuição das espécies para cada fator, testemunho KF-C.

Table 8. Score matrix with the contribution of the species for each factor, core KF-C.

\begin{tabular}{|c|c|c|}
\hline \multirow{2}{*}{$\begin{array}{l}\text { Testemunho KF-C } \\
\text { Espécies }\end{array}$} & \multicolumn{2}{|c|}{ Escores } \\
\hline & Fator 1 & Fator 2 \\
\hline O. universa & 0,0208 & $-0,4444$ \\
\hline G. conglobatus & $-0,1962$ & $-0,1185$ \\
\hline G. ruber (var. rosa) & 0,1301 & $-0,0350$ \\
\hline G. ruber (var. branca) & 4,8456 & 1,9498 \\
\hline G. tenella & $-0,3629$ & $-0,0067$ \\
\hline G. sacculifer (s/ saco) & $-1,560$ & 4,2933 \\
\hline G. sacculifer (c/ saco) & $-0,1994$ & 0,0650 \\
\hline S. dehiscens & $-0,0206$ & $-0,4855$ \\
\hline G. siphonifera & 0,2277 & $-0,1150$ \\
\hline G. calida & $-0,0537$ & $-0,2037$ \\
\hline G. bulloides & $-0,0576$ & $-0,4262$ \\
\hline G. falconensis & $-0,6570$ & 0,4151 \\
\hline G. digitata & $-0,0128$ & $-0,4971$ \\
\hline G. rubescens & $-1,4642$ & 1,6900 \\
\hline N. dutertrei & 0,1340 & $-0,5639$ \\
\hline Pulleniatina sp. & $-0,0397$ & $-0,4211$ \\
\hline G. inflata & $-0,0081$ & $-0,4984$ \\
\hline G. truncatulinoides dex. & $-0,0958$ & $-0,1788$ \\
\hline G. truncatulinoides $\sin$. & $-0,0088$ & $-0,4916$ \\
\hline G. crassaformis & 0,0675 & $-0,5229$ \\
\hline G. hirsuta & $-0,0128$ & $-0,4971$ \\
\hline G. scitula & $-0,0697$ & $-0,3967$ \\
\hline G. menardii & $-0,0508$ & $-0,2912$ \\
\hline G. tumida flexuosa & $-0,1616$ & $-0,2414$ \\
\hline G. tumida & $-0,3236$ & 0,0041 \\
\hline C. nitida & 0,1584 & $-0,5077$ \\
\hline G. glutinata & $-0,1578$ & $-0,0217$ \\
\hline T. humilis & $-0,0451$ & $-0,4585$ \\
\hline H. pelagica & $-0,0128$ & $-0,4971$ \\
\hline
\end{tabular}

Tabela 9. Comunalidades e cargas fatoriais, testemunho KF-C.

Table 9. Commonalities and factor loadings, core KF-C.

\begin{tabular}{cccc}
\hline \multicolumn{2}{c}{ Testemunho KF-C } & \multicolumn{2}{c}{ Carga fatorial } \\
\hline Amostra $(\mathrm{cm})$ & Comunalidade & Fator 1 & Fator 2 \\
\hline 0 & 0,97 & 0,7545 & 0,6402 \\
11 & 0,99 & 0,7998 & 0,5939 \\
23 & 0,99 & 0,7434 & 0,6627 \\
35 & 0,99 & 0,7421 & 0,6831 \\
46 & 0,99 & 0,6856 & 0,7214 \\
53 & 0,98 & 0,5291 & 0,8414 \\
57 & 0,98 & 0,6791 & 0,7257 \\
61 & 0,99 & 0,8368 & 0,5391 \\
65 & 0,99 & 0,7897 & 0,6087 \\
69 & 0,99 & 0,7353 & 0,6751 \\
74 & 0,99 & 0,7541 & 0,6519 \\
81 & 0,99 & 0,7687 & 0,6374 \\
92 & 0,99 & 0,8130 & 0,5777 \\
103 & 0,98 & 0,6945 & 0,7098 \\
114 & 0,99 & 0,6316 & 0,7698 \\
\hline
\end{tabular}


expressam a proporção dos dois fatores em cada amostra do testemunho KF-C. A comunalidade muito elevada, sempre acima de 0,90 , confere alto grau de confiabilidade na análise de todas as amostras (Toledo et al., 1999).

Como destacado na Tabela 9 , a amostra $61 \mathrm{~cm}$ caracteriza o fator 1 , já que exibe a maior carga do fator $1(0,8368)$ e a menor carga fatorial do fator $2(0,5391)$. A amostra $53 \mathrm{~cm}$ foi a amostra padrão do fator 2, pois apresentou a maior carga para este fator $(0,8414)$ e a menor carga fatorial para o fator $1(0,5291)$. A Figura 3 apresenta os histogramas dos fatores 1 e 2 e as espécies analisadas. $O$ fator 1, demonstra o domínio da espécie Globigerinoides ruber 'branca' (G. ruber W), enquanto o fator 2 exibe o domínio de Globigerinoides sacculifer 's/ saco' (G. sacc. SS).

Testemunho KF-B. A análise fatorial modo-Q para o testemunho KF-B apresentou resultados bastante similares, e a interpretação dos fatores seguiu o mesmo padrão apresentado para o KF-C. Neste caso, foram extraídos oito fatores, cuja variância total acumulada atingiu $100 \%$ e, assim como na análise anterior, apenas o primeiro fator teve autovalor maior que 1,0 (Tabela 10). Portanto, o fator 1 foi suficiente para explicar $97,18 \%$ da variância dos dados ao longo do tempo e, por isto, deve ser considerado como o principal parâmetro ambiental determinante para as assembleias dos foraminíferos planctônicos aqui estudadas.

Apesar de exibir autovalor muito baixo e percentual pouco significativo, o fator 2 foi considerado na interpretação do testemunho KF-B. Da mesma forma que no testemunho KF$\mathrm{C}$, sua influência foi secundária, porém, juntos os dois fatores explicam $99,47 \%$ da variabilidade total dos dados. Assim como no KF-C, nos dados da matriz de escores gerada pela análise fatorial, observa-se que o fator 1 é completamente dominado pela espécie Globigerinoides ruber 'branca' $(4,9218)$. Para o fator 2 verificam-se os maiores escores para Globigerinoides sacculifer 's/ saco' $(4,1255)$ (Tabela 11). A matriz de escores aponta quanto cada espécie contribuiu para cada um dos fatores extraídos.

A Tabela 12 exibe as comunalidades e as cargas fatoriais dos fatores 1 e 2 . Assim como no KF-C, os valores de comunalidade se mantiveram sempre muito elevados, acima de 0,90 . Nesta tabela é possível verificar que a amostra 1 $\mathrm{cm}$ caracteriza o fator 1 , já que exibe a maior carga do fator $1(0,8566)$ e a menor carga fatorial do fator $2(0,5095)$. A amostra $19 \mathrm{~cm}$ foi a amostra padrão do fator 2 , pois apresentou a maior carga para este fator $(0,8651)$ e a menor carga fatorial para o fator $1(0,5002)$.

Fator 1 (KF-C)

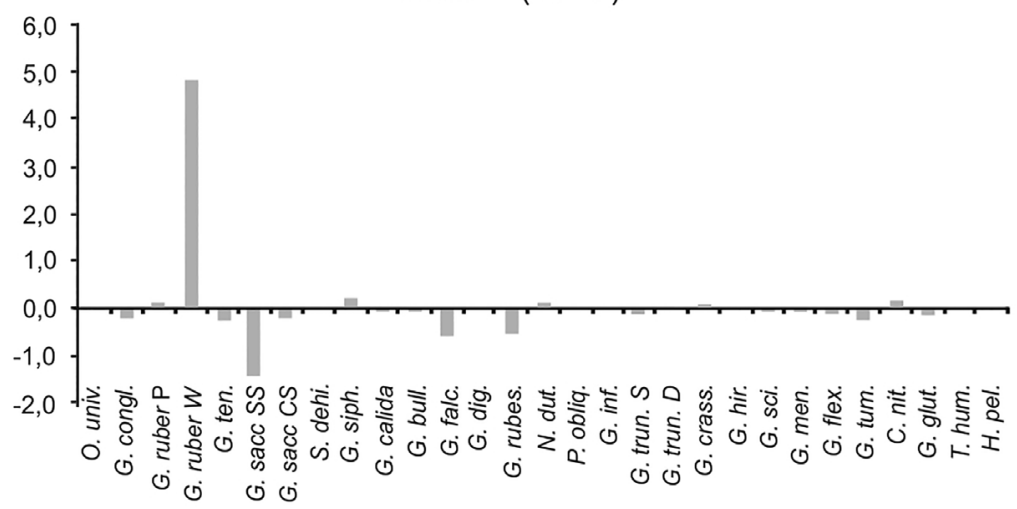

Fator $2(\mathrm{KF}-\mathrm{C})$

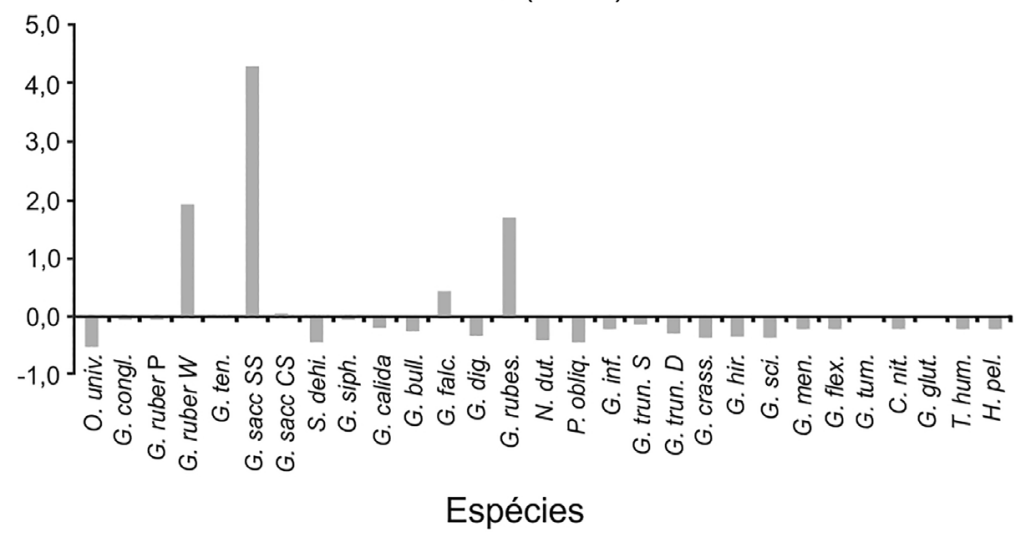

Figura 3. Histogramas demonstrando a contribuição das espécies de foraminíferos planctônicos para os fatores 1 e 2, testemunho KF-C.

Figure 3. Histogram showing the contribution of planktonic foraminifera to the factors 1 and 2, core KF-C. 
Tabela 10. Fatores, autovalores e suas variâncias para o testemunho KF-B.

Table 10. Factors, eigenvalues and total variance for the core KF-B.

\begin{tabular}{cccc}
\hline \multicolumn{3}{c}{ Testemunho KF-B } \\
\hline Fatores & Autovalor & Variância (\%) & Variância total acumulada (\%) \\
\hline 1 & 7,77 & 97,18 & 97,18 \\
2 & 0,18 & 2,28 & 99,47 \\
3 & 0,02 & 0,28 & 99,75 \\
4 & 0,01 & 0,14 & 99,88 \\
5 & 0,003 & 0,03 & 99,92 \\
6 & 0,002 & 0,03 & 99,95 \\
7 & 0,002 & 0,02 & 99,98 \\
8 & 0,001 & 0,01 & 100 \\
\hline
\end{tabular}

Tabela 11. Matriz de escores com a contribuição das espécies para cada fator, testemunho KF-B.

Table 11. Score matrix with the contribution of the species for each factor, core KF-B.

\begin{tabular}{|c|c|c|}
\hline \multirow{2}{*}{$\begin{array}{l}\text { Testemunho KF-B } \\
\text { Espécies }\end{array}$} & \multicolumn{2}{|c|}{ Escores } \\
\hline & Fator 1 & Fator 2 \\
\hline O. universa & 0,0674 & $-0,4200$ \\
\hline G. conglobatus & $-0,1598$ & $-0,2409$ \\
\hline G. ruber (var. rosa) & 0,1842 & 1,9172 \\
\hline G. ruber (var. branca) & 4,9218 & 1,9983 \\
\hline G. tenella & $-0,1951$ & $-0,0964$ \\
\hline G. sacculifer (s/ saco) & $-2,2041$ & 4,1255 \\
\hline G. sacculifer (c/ saco) & $-0,6467$ & 1,3805 \\
\hline S. dehiscens & $-0,1396$ & $-0,3948$ \\
\hline G. siphonifera & 0,3608 & $-0,4071$ \\
\hline G. calida & 0,6467 & $-0,8045$ \\
\hline G. bulloides & $-0,2971$ & $-0,0843$ \\
\hline G. falconensis & $-0,2010$ & 0,1292 \\
\hline G. digitata & $-0,1740$ & $-0,3672$ \\
\hline G. rubescens & $-0,1079$ & $-0,2077$ \\
\hline T. humilis & $-0,1332$ & $-0,4204$ \\
\hline N. dutertrei & $-0,1332$ & $-0,4204$ \\
\hline Pulleniatina sp. & $-0,4242$ & 0,0649 \\
\hline G. inflata & $-0,2164$ & $-0,2312$ \\
\hline G. truncatulinoides dex. & $-0,3316$ & 0,1043 \\
\hline G. truncatulinoides $\sin$. & $-0,1349$ & $-0,3825$ \\
\hline G. crassaformis & $-0,0937$ & $-0,4514$ \\
\hline G. hirsuta & $-0,2529$ & $-0,2220$ \\
\hline G. scitula & $-0,1136$ & $-0,3914$ \\
\hline G. menardii & $-0,0774$ & $-0,4240$ \\
\hline G. tumida flexuosa & 0,1159 & $-0,6175$ \\
\hline G. tumida & 0,0109 & $-0,5193$ \\
\hline C. nitida & 0,1128 & $-0,5405$ \\
\hline G. glutinata & 0,2277 & $-0,5311$ \\
\hline H. pelagica & $-0,1332$ & $-0,4204$ \\
\hline H. digitata & $-0,1332$ & $-0,4204$ \\
\hline
\end{tabular}

Tabela 12. Comunalidades e cargas fatoriais, testemunho KF-B.

Table 12. Commonalities and factor loadings, core KF-B.

\begin{tabular}{cccc}
\hline \multicolumn{2}{c}{ Testemunho KF-B } & \multicolumn{2}{c}{ Carga fatorial } \\
\hline Amostra $(\mathrm{cm})$ & Comunalidade & Fator 1 & Fator 2 \\
\hline 1 & 0,99 & 0,8566 & 0,5095 \\
3,5 & 0,99 & 0,8386 & 0,5420 \\
6 & 0,98 & 0,7728 & 0,6224 \\
8,5 & 0,99 & 0,8044 & 0,5911 \\
10,5 & 0,99 & 0,7536 & 0,6544 \\
12,5 & 0,99 & 0,6951 & 0,7161 \\
15 & 0,99 & 0,6789 & 0,7307 \\
19 & 0,99 & 0,5002 & 0,8651 \\
\hline
\end{tabular}

A Figura 6 apresenta os histogramas dos fatores 1 e 2, respectivamente, e as espécies analisadas no KF-B. Assim como no KF-C, a figura representativa do fator 1 demonstra o domínio da espécie Globigerinoides ruber 'branca', e a figura representativa do fator 2 exibe o domínio da espécie Globigerinoides sacculifer 's/ saco'.

\section{DISCUSSÃO}

Com base nos resultados apresentados pela análise fatorial, o significado do fator $1 \mathrm{em}$ ambos os testemunhos está associado à espécie Globigerinoides ruber 'branca', conhecida por ocorrer em abundância na camada de mistura de regiões tropicais (Bé \& Tolderlund, 1971; Bé, 1977; Hemleben et al., 1989; Hilbrecht, 1996). Por este motivo, o fator 1 foi denominado fator camada de mistura. $\mathrm{O}$ fator 2 foi associado à espécie Globigerinoides sacculifer 's/ saco', tanto no testemunho KF-C como no KF-B, e então denominado fator temperatura superficial, pela conhecida relação entre a distribuição da espécie e este parâmetro (Hilbrecht, 1996).

A maior abundância relativa observada no testemunho KF-C foi da espécie Globigerinoides ruber 'branca', em 61 $\mathrm{cm}$ (15.300 anos). Concomitantemente, neste período foi observada a menor abundância relativa de Globigerinoides sacculifer 's/ saco'. A segunda espécie mais abundante, G. sacculifer 's/ saco', exibiu sua maior abundância relativa em $53 \mathrm{~cm}$ (13.300 anos). Simultaneamente, neste período foi observada a menor abundância relativa de G. ruber 'branca'.

Dentro do período estudado foi evidente a preponderância do fator 1 (Figuras 4,7), destacando a importância da dinâmica da camada de mistura nesta região. No testemunho KF-C o fator 1 é dominante ao longo do tempo (Figura 4), no entanto, nas amostras $53 \mathrm{~cm}$ e $46 \mathrm{~cm}$ (13.300-11.600 anos) o fator 2, temperatura superficial, parece ter exercido maior influência na distribuição dos foraminíferos planctônicos. No KF-B, a partir de 12,5 cm (24.000 anos), é evidente o aumento da abundância relativa de Globigerionoides ruber 'branca'. Antes desse momento foram observadas as maiores abundâncias relativas de Globigerinoides sacculifer 's/ saco' e, consequentemente, o predomínio do fator 2 sobre o fator 1 . Até 24.000 anos a temperatura superficial parece ter exercido maior influência.

A análise fatorial modo-Q representou verdadeiramente o comportamento das espécies de foraminíferos planctônicos 
KF-C

cargas fatoriais $\mathrm{x}$ profundidade

fator $1 \mathrm{x}$ fator 2

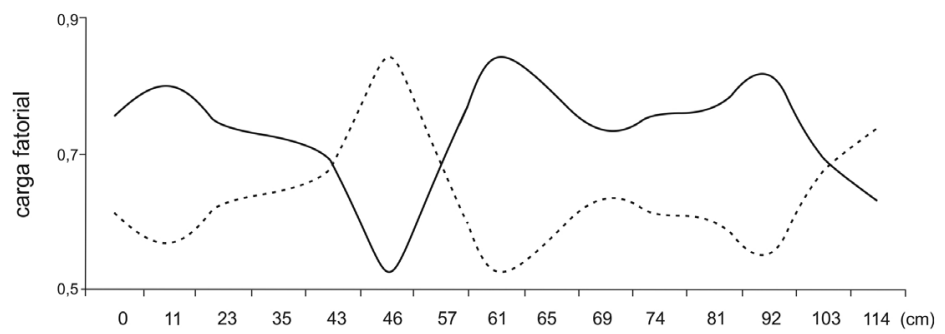

Figura 4. Comparação dos fatores 1 e 2 (modo-Q) em função de suas cargas fatoriais ao longo do testemunho KF-C.

Figure 4. Comparison of factors 1 and 2 (Q-mode) according to their factor loadings along core KF-C.

Cargas Fatoriais (KF-C)

fator $1 \mathrm{x}$ fator 2

Rotação: Varimax - Extração: Componentes Principais

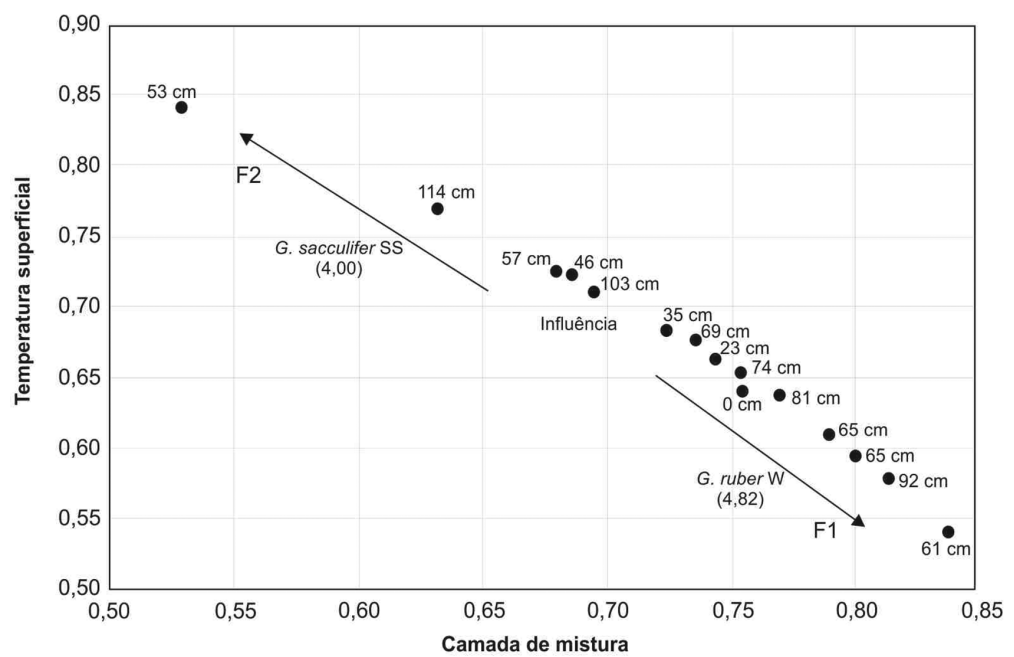

Figura 5. Projeção da correlação entre os fatores 1 e 2, testemunho KF-C.

Figure 5. Correlation projection between factors 1 and 2, core KF-C.

e sua relação com os parâmetros ambientais (camada de mistura e temperatura superficial marinhas). A atuação dos fatores na estruturação da assembleia de foraminíferos pode ser visualizada quando comparamos os fatores 1 e 2 ao longo do tempo (Figuras 4, 7).

É importante advertir que o fator temperatura superficial é secundário e explica apenas 1,12\% (KF-C) e 2,28\% (KF-B) da variância total. As zonas de influências obtidas do gráfico de correlação entre os fatores 1 e 2 permitem observar que a maior parte das amostras está próxima ao fator camada de mistura, caracterizando, desta forma, o maior peso deste fator para essa região (Figuras 5, 8). Somente duas amostras $(53 \mathrm{~cm}$ e $114 \mathrm{~cm}$ ) são fortemente influenciadas pelo fator 2, temperatura superficial, no testemunho KF-C (Figura 5) em 13.300 e 29.900 anos. As três amostras situadas entre os fatores 1 e $2(57 \mathrm{~cm}$, $46 \mathrm{~cm}$ e $103 \mathrm{~cm}$ ) no KF-C e a amostra $12,5 \mathrm{~cm}$ no KF-B, estão na zona de influência e sugerem uma mudança nas condições oceânicas atuantes nesses intervalos (Figuras 5,8).
De acordo com a estratigrafia isotópica, a amostra 103 $\mathrm{cm}$ data de, aproximadamente, 26.700 anos A.P., que poderia ser interpretada como o início do EIM 2. A amostra $12,5 \mathrm{~cm}$ $(=24.000$ anos A.P.) do testemunho KF-B é representativa do Último Máximo Glacial (UMG). O UMG foi caracterizado por uma intensa oscilação nos padrões oceânicos do Atlântico tropical oeste (Arz et al., 1998; Arz et al., 1999; Behling et al., 2000), representados neste estudo por valores mais positivos de $\delta^{18} \mathrm{O}$ (maior volume de gelo) e pela freqüência de espécies associadas a estas variações (Globorotalia truncatulinoides e $G$. inflata). A amostra $57 \mathrm{~cm}$ (= 14.300 anos) marca a transição do EIM 2 para o EIM 1. A amostra $46 \mathrm{~cm}$, com idade estimada em 11.600 anos, pode estar associada ao evento climático de curta duração do hemisfério norte, o Younger Dryas. Estes eventos são, realmente, caracterizados por intensa oscilação nos padrões oceânicos devido à instabilidade climática (Jennerjahn et al., 2004; Weldeab et al., 2006). A detecção destes intervalos pela análise estatística reforça sua importante influência no sistema. 
Fator 1 (KF-B)

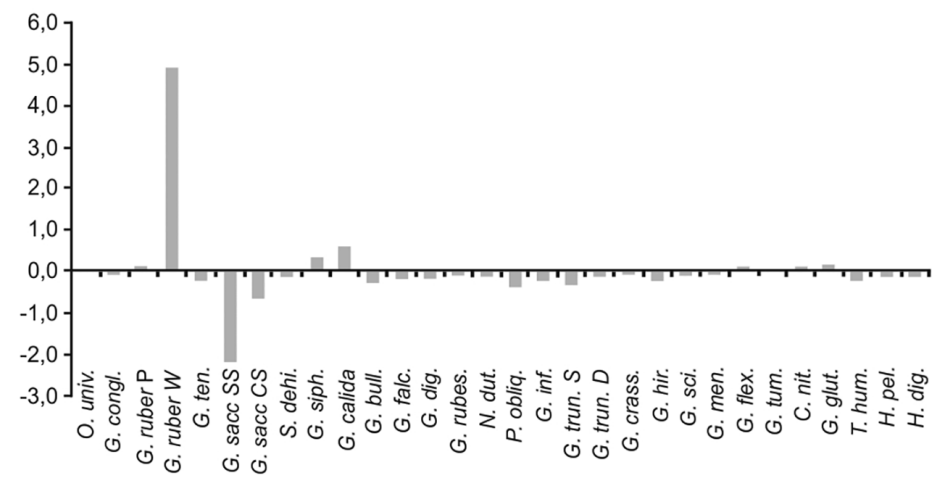

Fator $2(\mathrm{KF}-\mathrm{B})$

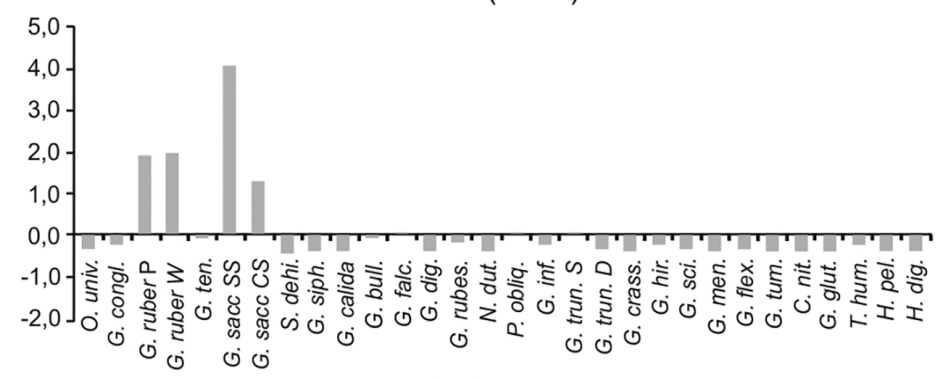

Espécies

Figura 6. Histogramas demonstrando a contribuição das espécies de foraminíferos planctônicos para os fatores 1 e 2, testemunho KF-B.

Figure 6. Histogram showing the contribution of planktonic foraminifera to the factors 1 and 2, core KF-B.

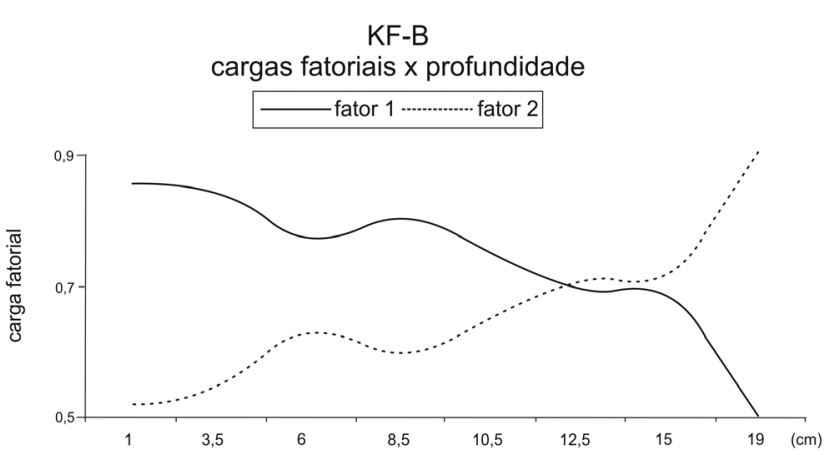

Figura 7. Comparação dos fatores 1 e 2 (modo-Q) em função de suas cargas fatoriais ao longo do testemunho KF-B.

Figure 7. Comparison of factors 1 and 2 (Q-mode) according to their factor loadings along core KF-B.

Com base no resultado evidenciado pelo fator 1 (camada de mistura) foi possível inferir que durante o EIM 2 a camada de mistura era menos espessa devido à coluna d'água mais turbulenta e menos estratificada. Acredita-se que isto seja devido a maior intensidade dos ventos alísios de SE neste período. A magnitude dos ventos alísios teria intensificado a Corrente Sul Equatorial aumentando, desta forma, o transporte de massa e calor da região subtropical leste para a região tropical oeste do Atlântico Sul. O empilhamento de águas nesta porção do Atlântico teria aumentado a profundidade da termoclina e da nutriclina, deslocando-a para maiores profundidades. Neste sentido, esta porção do Atlântico tropical oeste teria atuado como um reservatório de calor durante o EIM 2 e na transição EIM 2-EIM 1, o que está de acordo com estudos anteriores (Toledo et al., 2007; Weldeab et al., 2006; Kim \& Schneider, 2003).

O fator 2 (temperatura superficial), por outro lado, indicou que, assim como no EIM 2, durante o evento de resfriamento de curta duração no hemisfério norte (Younger Dryas), o Atlântico tropical oeste exibiu um discreto aquecimento de suas águas superficiais, provocando a diminuição do transporte de massa através do equador. $\mathrm{O}$ enfraquecimento da circulação termohalina, pode ter levado à estagnação e ao acúmulo de sal e calor durante estes eventos no Atlântico tropical oeste, conforme observado por Kim \& Schneider (2003).

Este estudo demonstrou que a partir do EIM 1 o Atlântico tropical oeste teve características mais amenas, relacionadas, principalmente, a menor intensidade dos ventos alísios de SE e ao deslocamento da Zona de Convergência Intertropical (ZCIT) para o hemisfério sul. O deslocamento da ZCIT para sul teria levado consigo as maiores temperaturas superficiais. Isto resultaria em uma camada superficial menos densa e mais estável. 


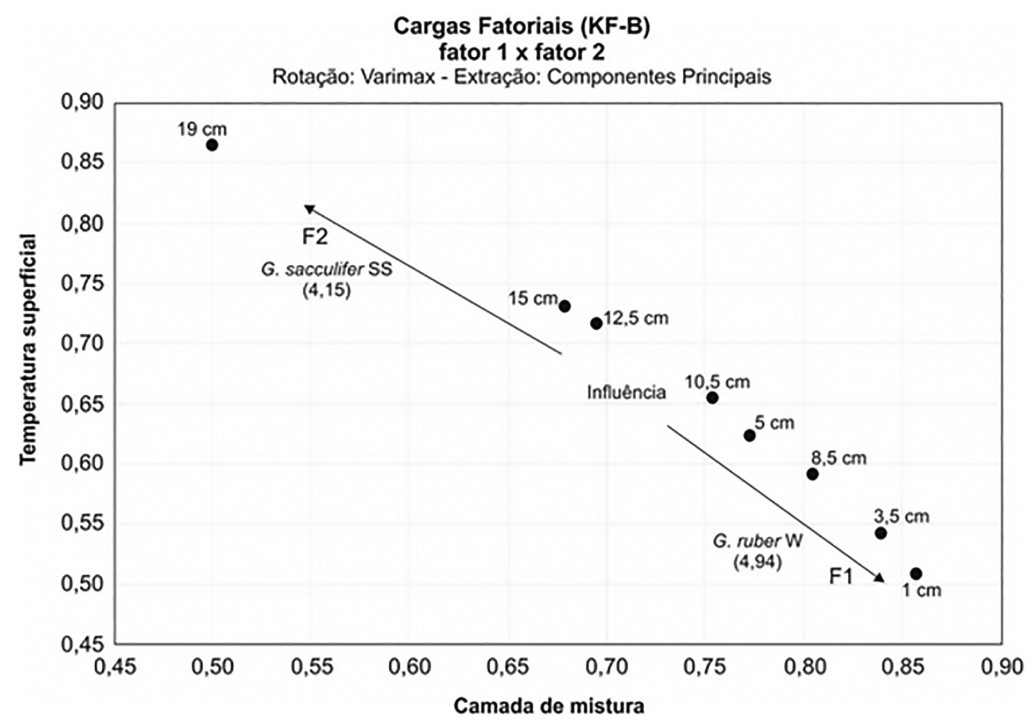

Figura 8. Projeção da correlação entre os fatores 1 e 2, testemunho KF-B.

Figure 8. Correlation projection between factors 1 and 2, core KF-B.

\section{CONCLUSÕES}

A partir da análise da estratigrafia isotópica do oxigênio $\left(\delta^{18} \mathrm{O}\right)$ foi possível reconhecer que ambos os testemunhos estudados alcançaram o EIM 2. O EIM 1 está bem representado no testemunho KF-C, porém no KF-B apenas duas amostras são indicativas deste estágio. As informações obtidas da análise faunística e da análise multivariada são complementares. A análise faunística caracterizou a assembleia de foraminíferos planctônicos como típica associação tropical, constituída por espécies de águas quentes dominando durante os últimos 30.000 anos. Os resultados deste estudo indicaram uma relativa estabilidade das condições ambientais gerais ao longo deste período na área de estudo. A análise multivariada corroborou a análise faunística e facilitou o reconhecimento da relação existente entre os parâmetros ambientais determinantes e a distribuição temporal das espécies dominantes. A análise fatorial modo-Q detectou apenas dois fatores envolvidos na dinâmica temporal dos foraminíferos planctônicos. Estes fatores foram interpretados como variações na camada de mistura e na temperatura da superfície do mar, respectivamente fator 1 e fator 2 , como as principais condições oceanográficas capazes de influenciar a distribuição dos foraminíferos planctônicos nesta região. A razão pela qual apenas um dos fatores (camada de mistura) compõe $97 \%$ da variância dos dados, corrobora a estabilidade ambiental apontada pela análise faunística. Isto indica que ocorreu pouca variação, mas quando houve foi determinada por alteração basicamente em um único fator. Durante os últimos 30.000 anos os eventos de instabilidade na camada de mistura foram determinantes na dinâmica populacional dos foraminíferos planctônicos. A análise fatorial modo-Q facilitou o reconhecimento dos padrões de distribuição destes microfósseis ao longo do tempo e suas afinidades com os parâmetros ambientais inerentes ao ambiente marinho.

\section{REFERÊNCIAS}

Andreasen, D.J. \& Ravelo, A.C. 1997. Tropical Pacific Ocean thermocline depth reconstructions for the last glacial maximum. Paleoceanography, 12:395-413. doi:10.1029/97PA00822

Arz, H.W.; Pätzold, J. \& Wefer, G. 1998. Correlated millenial-scale changes in surface hydrography and terrigenous sediment yield inferred from last-glacial marine deposits off northeastern Brazil. Quaternary Research, 50:157-166. doi:10.1006/qres.1998.1992

Arz, H.W.; Pätzold, J. \& Wefer, G. 1999. Climatic changes during the last deglaciation recorded in sediment cores form the northeastern Brazilian Continental Margin. Geo-Marine Letters, 19:209-218. doi:10.1007/s003670050111

Behling, H.; Arz, H.W.; Pätzold, J. \& Wefer, G. 2000. Late Quaternary vegetational and climate dynamics in northeastern Brazil, inferences from marine core GeoB 3104-1. Quaternary Science Reviews, 19:981-994. doi:10.1016/S0277-3791(99)00046-3

Bolli, H.M. \& Saunders, J.B. 1985. Oligocene to Holocene low latitude planktic foraminifera. In: H.M. Bolli; J.B. Saunders \& K. Perch-Nielsen (eds.) Plankton Stratigraphy, Cambridge University Press, p. 156-262.

Broecker, W.S. \& Takahashi, T. 1978. The relationship between lysocline depth and in situ carbonate ion concentration. Deep Sea Research Part I: Oceanographic Research Papers, 25:65-95.

Buccianti, A. \& Esposito, P. 2004. Insights into Late Quaternary calcareous nannoplankton assemblages under the theory of statistical analysis for compositional data. Palaeogeography, Palaeoclimatology, Palaeoecology, 202:209-227. doi:10.1016/ S0031-0182(03)00635-7

Butzin, M.; Prange, M. \& Lohmann, G. 2005. Radiocarbon simulations for the glacial ocean: the effects of wind stress, Southern Ocean sea ice and Heinrich events. Earth and Planetary Science Letters, 235:45-61. doi:10.1016/j.epsl.2005.03.003

Chen, M.-T. \& Prell, W.L. 1998. Faunal distribution patterns of planktonic foraminifers in surface sediments of the low-latitude Pacific. Palaeogeography, Palaeoclimatology, Palaeoecology, 137:55-77. doi:10.1016/S0031-0182(97)00095-3 
Costa, K.B. 2000. Variações paleoceanográficas na porção oeste do Atlântico Sul entre o Último Máximo Glacial e o Holoceno: isótopos estáveis de oxigênio e carbono e a razão $\mathrm{Cd} / \mathrm{Ca}$ em foraminiferos bentônicos. Programa de Pós-graduação em Geociências, Universidade Federal do Rio Grande do Sul, Tese de Doutorado, $236 \mathrm{p}$.

Ericson, D.B. \& Wollin, G. 1968. Pleistocene climates and chronology in deep-sea sediments. Science, 162:1227-1234. doi:10.1126/science.162.3859.1227

Fairbanks, R.G.; Mortlock, R.A.; Chiu, T.-C.; Cao, L.; Kaplan, A.; Guilderson, T.P.; Fairbanks, T.W. \& Bloom, A.L. 2005. Radiocarbon calibration curve spanning 10,000 to 50,000 years B.P. based on paired ${ }^{230} \mathrm{Th} /{ }^{234} \mathrm{U} /{ }^{238} \mathrm{U}$ and ${ }^{14} \mathrm{C}$ dates on pristine corals. Quaternary Science Reviews, 24:1781-1796. doi:10.1016/j.quascirev.2005.04.007

Frenz, M. \& Henrich, R. 2007. Carbonate dissolution revealed by silt grain-size distribution: comparison of Holocene and Last Glacial Maximum sediments from the pelagic South Atlantic. Sedimentology, 54:391-404. doi:10.1111/j.13653091.2006.00841.x

Gandhi, M.S. \& Solai, A. 2010. Statistical studies and ecology of benthic foraminifera from the depositional environment: a case study between Mandapam and Tuticorin, South East Coast of India. International Journal of Research and Reviews in Applied Sciences, 5:86-94.

Guerreiro, C.; Cachão, M.; De Stigter, H.; Oliveira, A. \& Rodrigues, A. 2011. Compositional data analysis as a potential tool to study the (paleo)ecology of calcareous nannoplankton from the Central Portuguese submarine canyons (W off Portugal). In: INTERNATIONAL WORKSHOP ON COMPOSITIONAL DATA ANALYSIS, 4, 2011. Proceedings, Girona, Universitat de Girona, p.1-12.

Hemleben, C.; Spindler, M. \& Anderson, O.R. 1989. Modern Planktonic Foraminifera. New York, Springer, 363 p.

Hilbrecht, H. 1996. Extant planktic foraminifera and the physical environment in the Atlantic and Indian Oceans. Mitteilungen aus dem Geologischen Institut der Eidgen, Technischen Hochschule und der Universität Zürich, 93 p.

Hirama, M.V.; Toledo, F.A.L.; Camillo Jr., E.; Costa, K.B. \& Quadros, J.P. 2010. Q-Mode and R-Mode factor analysis in quantitative studies of microfossils of the late Quaternary in sediments from the Brazilian continental margin. Terrae, 7:41-49.

Jayalakshmy, K.V. \& Rao K.K. 2003. Multivariate statistical study with a factor analysis of foraminiferal fauna from the Chilka Lake, India. Journal of the Marine Biological Association of India, 45:133-143.

Jennerjahn, T.C.; Ittekkot, V.; Arz, H.W.; Behling, H.; Pätzold, J. \& Wefer, G. 2004. Asynchronous terrestrial and marine signals of climate change during Heinrich events. Science, 306:2236-2239. doi:10.1126/science. 1102490

Kemle-Von Mucke, S. \& Oberhänsli, H. 1999. The distribution of living planktic foraminifera in relation to Southeast Atlantic oceanography. In: G. Fischer \& G. Wefer (eds.) Use of proxies in paleoceanography: examples from the South Atlantic, SpringerVerlag, p. 91-115.

Kim, J.-H. \& Schneider, R.R. 2003. Low-latitude control of interhemispheric sea-surface temperature contrast in the tropical Atlantic over the past 21 k years: the possible role of SE trade winds. Climate Dynamics, 21:337-347. doi:10.1007/s00382-003-0341-5

Kucera, M. \& Malmgren, B.A. 1998. Log ratio transformation of compositional data - a resolution of the constant sum constraint. Marine Micropaleontology, 34:117-120. doi:10.1016/S03778398(97)00047-9
Lisiecki, L.E. \& Raymo, M.E. 2005. A Pliocene-Pleistocene stack of 57 globally distributed benthic $\delta^{18} \mathrm{O}$ records. Paleoceanography, 20:PA2007. doi:10.1029/2004PA001071

Mix, A.C. 1987. The oxygen-isotope record of glaciation. In: W.F. Ruddiman \& H.E. Wright (eds.) The geology of North America, North America and adjacent oceans during the last deglaciation, Geological Society of America, p.111-135.

Molfino, B.; Kipp, N.G. \& Morley, J. 1982. Comparison of foraminiferal, coccolithophorid, and radiolarian paleotemperature equations: assemblage coherency and estimate concordancy. Quaternary Research, 17:279-313.

Oberhänsli, H.; Bénier, C.; Meinecke, G.; Schmidt, H.; Schneider, R. \& Wefer, G. 1992. Planktonic foraminifers as tracers of ocean currents in the eastern South Atlantic. Paleoceanography, 7:607632. doi:10.1029/92PA01236

Oláyíwolá, M.A. \& M. Odébòdé, O. 2011. Multivariate analysis of planktonic foraminiferal assemblage of Southwestern Nigerians surface continental shelves' sediments. European Journal of Scientific Research, 54:84-101.

Parker, W.C. \& Arnold, A.J. 1999. Quantitative methods of data analysis in foraminiferal ecology. In: B.K.S. Gupta (ed.) Modern Foraminifera, Academic Publishers, p. 71-89.

Peterson, R.G. \& Stramma, L. 1991. Upper-level circulation in the South Atlantic. Proceedings in Oceanography, 26:1-73. doi:10.1016/0079-6611(91)90006-8

Prentice, I.C. 1986. Multivariate methods for data analysis. In: B.E. Berglund (ed.) Handbook of Holocene Palaeoecology and Palaeohydrology, Willey, p. 775-797.

Ravelo, A.C.; Fairbanks, R.G. \& Philander, S.G.H. 1990 Reconstructing tropical Atlantic hydrography using planktonic foraminifera and an ocean model. Paleoceanography, 5:409-431. doi:10.1029/PA005i003p00409

Thompson, P.R.; Be, A.W.H.; Duplessy, J.C. \& Shackleton, N.J. 1979. Disappearance of pink-pigmented Globigerinoides ruber at $120,000 \mathrm{yr}$ BP in the Indian and Pacific oceans. Nature, 280:554-558. doi:10.1038/280554a0

Toledo, F.A.L. 2000. Variações paleoceanográficas nos últimos 30.000 anos no oeste do Atlântico Sul: isótopos de oxigênio, assembleia de foraminíferos planctônicos e nanofósseis calcários. Programa de Pós-Graduação em Geociências, Universidade Federal do Rio Grande do Sul, Tese de Doutorado, $245 \mathrm{p}$.

Toledo, F.A.L.; Ayup-Zouain, R.N. \& Costa, K.C. 1999. Análise fatorial (modo-Q) em estudos quantitativos de nanofósseis calcários do Quaternário superior em um testemunho da Bacia de Campos, RJ-Brasil. Pesquisas, 26:62-72.

Toledo, F.A.L.; Costa, K.B. \& Pivel, M.A.G. 2007. Salinity changes in the western tropical South Atlantic during the last $30 \mathrm{kyr}$. Global and Planetary Change, 57:383-395. doi:10.1016/j. gloplacha.2007.01.001

Ufkes, E.; Jansen, J.H.F. \& Brummer, G.J. 1998. Living planktonic foraminifera in the eastern South Atlantic during spring: indicators of water masses, upwelling and Congo (Zaire) River plume. Marine Micropaleontology, 33:27-53. doi:10.1016/ S0377-8398(97)00032-7

Weldeab, S.; Schneider, R.R. \& Kölling, M. 2006. Deglacial sea surface temperature and salinity increase in the Western Tropical Atlantic in synchrony with high latitude climate instabilities. Earth and Planetary Science Letters, 241:699-706. doi:10.1016/j.epsl.2005.11.012

Received in July, 2014; accepted in February, 2015. 